\title{
Emociones, creencias y actitudes del personal asistencial hacia habitantes de calle que usan drogas en Medellín, Colombia*
}

\author{
Emotions, beliefs and attitudes of healthcare workers towards homeless who use \\ drugs in Medellín, Colombia
}

\section{Emoções, crenças e atitudes do pessoal de assistência social com os moradores de rua que usam drogas em Medellín, Colômbia}

Maricelly Gómez Vargas'; Eliana María Hernández Ramírez; María Jimena Osorio Salazar; Andrés Felipe Tirado Otálvaro4; José Silverio Espinal Bedoya5; Daniel Ricardo Zaraza Morales; ; Lucas Dávila Cañas7; Luz Merly Saldarriaga Agudelo8

1 Magíster en Psicología. Universidad Católica Luis Amigó, Medellín, Colombia. maricelly.gomezva@amigo.edu.co. ORCID: https://orcid. org/0000-0002-2389-7821

2 Magíster Salud Mental. Universidad de Antioquia, Medellín, Colombia. emaria.hernandez@udea.edu.co. oRciD: https://orcid.org/00000001-8620-6496

3 Magíster en Intervenciones Psicosociales. Fundación Universitaria Claretiana. Medellín, Colombia. jimeos29@gmail.com. ORciD: https://orcid.org/0000-0002-0715-7348

4 Doctor en Salud Pública. Universidad Pontificia Bolivariana, Medellín, Colombia. felipe.tirado@upb.edu.co. ORCiD: https://orcid. org/0000-0001-9010-1494

5 Psicólogo. Corporación Surgir. Medellín, Colombia. silverio@corporacionsurgir.org. ORCID: https://orcid.org/0000-0003-0364-0487

6 Magíster en Salud Mental. Universidad Pontificia Bolivariana, Medellín, Colombia. danielzaraza@gmail.com. ORCID: https://orcid. org/0000-0002-4197-3787

7 Magíster en intervenciones psicosociales. Universidad Católica Luis Amigó, Medellín, Colombia. lucas.davilaca@amigo.edu.co. ORCID: https://orcid.org/0000-0002-5155-0868

8 Magíster en Salud Mental. Universidad de Antioquia, Medellín, Colombia. merly.saldarriaga@udea.edu.co. orcid: https://orcid. org/0000-0002-1993-890X

Recibido: 18/12/2020. Aprobado: 26/07/2021. Publicado: 9/08/2021

Gómez-Vargas M, Hernández Ramírez EM, Osorio-Salazar MJ, Tirado-Otálvaro AF, Espinal-Bedoya JS, Zaraza-Morales DR, Dávila-Cañas L, Saldarriaga-Agudelo LM. Emociones, creencias y actitudes del personal asistencial hacia habitantes de calle que usan drogas en Medellín, Colombia. Rev. Fac. Nac. Salud Pública. 2021;39(3):e344658. Dor: https://doi.org/10.17533/udea. rfnsp.e344658

* Este texto es producto de la investigación "Estudio multicéntrico sobre la reducción del estigma asociado al uso de drogas (Proyecto Colombia)", avalado por la Universidad Pontificia Bolivariana, sede Medellín. Radicado número 106C-05/18-38. Fecha de inicio: julio 2019. Fecha de finalización: julio 2021. Este proyecto se adscribe al estudio multicéntrico sobre reducción del estigma hacia personas que usan drogas, realizado por la Red Latinoamericana de Investigación sobre Estigma y Drogas, y liderado por la Universidad Federal de Juiz de Fora, de Brasil. 


\section{Resumen}

Objetivo: Identificar algunos aspectos relacionados con el estigma de personal asistencial hacia habitantes de calle consumidores de sustancias psicoactivas. Método: Para lograr este objetivo, se llevó a cabo un estudio cualitativo, con enfoque hermenéutico y de alcance descriptivo, mediante el análisis categorial de las transcripciones de las grabaciones de los discursos de dos grupos focales con 32 participantes, miembros del Sistema de Atención al Habitante de Calle Adulto de Medellín, Colombia. La sistematización y la codificación del análisis se hicieron en un archivo de Excel ${ }^{\circledR}$, destacándose la emergencia de las categorías: "emociones", "creencias" y "actitudes". Resultados: En los discursos de los participantes, se reiteran contenidos ambivalentes de los estigmas hacia esta población. Por una parte, la frustración en su trabajo, como principal emoción que coincide con las creencias de desconfianza y actitudes evasivas hacia ellos; $\mathrm{y}$, por otro, los sentimientos de gratificación por la labor realizada, acordes con posturas más protectoras y pacientes durante el acompañamiento, pero que no se alejan de un ideal moralista de cero consumos. Conclusión: Reconocer y discutir abiertamente sobre el estigma podría orientar otras formas más humanizadas en la labor con este tipo de población, en tanto se devela que el rol profesional no nos exime del prejuicio, el estereotipo y la discriminación.

-------Palabras clave: estereotipo, estigma, grupos profesionales, personas sin hogar, trastornos relacionados con sustancias psicoactivas.

\section{Abstract}

Objective: To identify some aspects related to the stigma of healthcare workers towards the homeless who consume psychoactive substances. Methodology: To achieve this objective, a qualitative study with a hermeneutic approach and descriptive scope was carried out, through the categorical analysis of transcriptions of recorded speeches from two focus groups comprised of 32 participants, members of the System of Attention to the Homeless Adult in Medellín, Colombia. Systematization and analysis encoding were performed in an Excel ${ }^{\circledR}$ file, highlighting the presence of categories: "emotions", "beliefs" and "attitudes". Results: Ambivalent contents of the stigma towards this population are frequent in participant's speeches. On the one hand, the frustration about healthcare work, as the main emotion that coincides with the beliefs of distrust and evasive attitudes towards the homeless. On the other hand, the feelings of gratification for the work done and the presence of more protective and forbearing attitudes, but that do not alienate from the moralistic ideal of zero consumption. Conclusion: Recognizing and openly discussing stigma could guide more humanized ways of working with this type of population, as it is showed that the healthcare role does not exempt them from prejudice, stereotype and discrimination.

---------Keywords: Stereotype, stigma, professional groups, homeless, psychoactive substance-related disorders.

\section{Resumo}

Objetivo: Identificar alguns aspectos relacionados ao estigma do pessoal de assistência social com os moradores de rua consumidores de substâncias psicoativas. Metodologia: Para alcançar este objetivo, foi realizado um estudo qualitativo, com abordagem hermenêutica e âmbito descritivo, através da análise categorial das transcrições das gravações dos discursos de dois grupos focais com 32 participantes, membros do Sistema de Atenção ao Morador de Rua Adulto de Medellín, Colômbia. A sistematização e a codificação da análise foram feitas num arquivo Excel $\AA$, destacando-se a emergência das categorias: "emoções", "crenças" e "atitudes". Resultados: Os participantes reiteraram em suas falas, conteúdos ambivalentes dos estigmas dessa população. Por um lado, a frustração em seu trabalho, com emoção principal que coincide com as crenças de desconfiança e atitudes evasivas com eles; e por outro, os sentimentos de gratidão pelo trabalho realizado, de acordo com posturas mais protetoras e pacientes durante o acompanhamento, mas que não se distanciam de um ideal moralista de consumo zero. Conclusão: Reconhecer e discutir abertamente o estigma poderia orientar outras formas mais humanizadas no trabalho com este tipo de população, e ao mesmo tempo se revela que o compromisso profissional não os exime do preconceito, o estereótipo e a discriminação.

Palavras-chave: Estereótipo; Estigma; Grupos profissionais; Moradores de rua; Transtornos relacionados com substâncias psicoativas. 


\section{Introducción}

El consumo de sustancias psicoactivas (SPA) es considerado un fenómeno multicausal, que representa actualmente enormes retos para la sociedad. Parte de los riesgos identificados en las personas que usan drogas (PUD) son la adicción, las enfermedades infecciosas y la exclusión social, esta última, dada principalmente por la estigmatización que, según Goffman [1], es un atributo social negativo que establece diferencias entre las personas que lo tienen y las que no, generando situaciones de rechazo y menosprecio hacia quienes poseen dicha condición. Goffman [1] afirma que el estigma ya era común entre los griegos, para referirse a los signos corporales que representaban un estatus moral socialmente denigrado.

Link y Phelan [2] plantean el estigma como el resultado de un proceso que involucra cinco componentes interrelacionados entre sí: en el primero, hay un etiquetamiento de la persona, debido a sus diferencias; en el segundo, la persona etiquetada es estereotipada de acuerdo con características indeseables; en el tercero, hay una separación entre "ellos" y "nosotros"; en el cuarto, y debido al etiquetamiento, se pierde la condición de sujeto, con la consecuente aparición de la discriminación, la devaluación, el rechazo y la exclusión; y, por último, en el quinto componente se involucra un ejercicio desigual del poder entre quienes tienen la etiqueta y los que no.

Otros autores señalan que el estigma es una secuencia de relaciones que involucra estereotipos, prejuicios y discriminación, añadiendo el concepto complejo estigma-discriminación, para darle al mismo una visión holística, pues sostienen que estos elementos constitutivos del estigma no se presentan de manera procesual, y sí como una articulación entre ellos, implicados de manera íntima, contextual y dinámica, como lo advierte la teoría de sistemas complejos [3,4].

En las PUD, el estigma es mayor al que se presenta por enfermedades como el sida, la depresión, la lepra o la esquizofrenia, razón por la cual son consideradas delincuentes o enfermas [5], al asociarse el consumo con conductas violentas y degradación humana. Esta situación despoja a las personas de todo rastro de humanidad, lo que dificulta un trato digno, aumenta su condición de vulnerabilidad y limita sus posibilidades de acceder a derechos fundamentales, como la salud, la educación y el trabajo [6,7]. Asimismo, las excluye de manera parcial o total de espacios de participación ciudadana y se enfrentan con barreras para acceder a beneficios sociales que les corresponde por ley [8], lo que las limita para encontrar y mantener vivienda, y afecta sus relaciones sociales y autoconcepto, entre otros efectos [9].

El estigma puede agravarse cuando las PUD, además, son habitantes de calle [10]. Según el estudio realizado por el Departamento Administrativo Nacional de Estadística en 2019, en Medellín y el Área Metropolitana se identificaron 3788 personas en esta condición. De ellas, el $9,5 \%$ dijo no consumir ningún tipo de sustancia, mientras que la mayoría de los habitantes de calle encuestados admitieron el consumo de varios tipos de drogas, siendo las más frecuentes el basuco o pasta base de cocaína $(66,1 \%)$, la marihuana (53\%), el alcohol (37,8\%), la cocaína (24,7\%) y los inhalantes tipo pegantes (15,9\%) [11].

Los profesionales sanitarios se constituyen en un grupo prioritario para acompañar a las PUD. Sin embargo, aquellos no están exentos de los prejuicios. La estigmatización es un fenómeno que no es ajeno a las personas que brindan algún tipo de atención a los habitantes de calle que usan drogas. En ellas también se dan estigmas, a pesar de haber tenido algún tipo de preparación para atenderlos y de haber elegido esta labor, incrementando el riesgo de realizar una atención inadecuada, debido al poco interés e insatisfacción al realizar su trabajo, descalificándolos, muchas veces, desde el trato, las actitudes o la palabra [5]. Es preciso señalar esto, por cuanto la salud pública se vería afectada cuando no se tienen en cuenta los diversos aspectos sociales, históricos e individuales que hacen parte de la práctica y vida cotidiana del personal asistencial que trabaja con consumidores de SPA, a quienes se les ha prometido atención humanizada en las políticas públicas.

Se ha evidenciado que el personal asistencial asume que el consumo de drogas obedece a defectos del carácter, responsabilizando a las PUD de su condición $[12,13]$. Al respecto, Ronzani, Higgins-Biddle y Furtado afirman que los enfermeros y los agentes comunitarios son el personal con el juicio moral más severo respecto al uso de sustancias [5]. Asimismo, en investigaciones realizadas en Colombia, España y Argentina, se encontró que los profesionales de la salud tienen un juicio estigmatizante hacia las PUD, basado en el desconocimiento de la multiplicidad de factores que condicionan la adicción, los vacíos en la formación que reciben sobre el tema en sus pregrados universitarios, la falta de sensibilización [13] y las experiencias individuales [14], lo que se traduce en desconfianza, deficiente prestación de los servicios [15-17] y poca motivación de los profesionales para realizar su labor, pues consideran que estas personas no son capaces de abandonar su condición [13].

Así, en este artículo se exponen los resultados del componente cualitativo de una investigación multicéntrica sobre reducción del estigma hacia habitantes de calle que usan drogas, por parte de personal asistencial que labora en el Sistema de Atención al Habitante de Calle de Medellín, y cuyo objetivo fue identificar algunos aspectos relacionados con el estigma de personal asistencial hacia habitantes de calle consumidores de sustancias psicoactivas. 


\section{Metodología}

En este apartado describimos la orientación epistemológica, teórica y metodológica. Visibilizamos el proceso indicando el tipo de participantes y la forma como llevamos a cabo la recolección e interpretación de las informaciones, siendo también relevante dejar claro nuestras consideraciones éticas.

\section{Tipo de estudio y enfoque}

Se realizó una investigación cualitativa, con enfoque hermenéutico y alcance descriptivo, sobre algunos aspectos relacionados con el estigma de personal asistencial pertenecientes al Sistema de Atención al Habitante de Calle Adulto de la ciudad de Medellín hacia habitantes de calle consumidores de sustancias psicoactivas.

Entendimos lo hermenéutico como un relato construido a partir de la voz de los participantes, para ser interpretado por el grupo de investigación, y ser luego triangulado con la literatura científica sobre el tema [18].

Este estudio hace parte de una investigación multicéntrica y cuasiexperimental de tipo pre-post, ejecutada por la Red Latinoamericana de Investigación sobre Estigma y Drogas, que se lleva a cabo en Colombia, Brasil y Uruguay, cuyo objetivo es evaluar una intervención psicológica con técnicas vivenciales para la reducción del estigma de los profesionales sanitarios hacia las PUD.

Para este artículo se presentan los análisis cualitativos de las conversaciones grabadas durante los talleres realizados en Medellín, Colombia.

\section{Participantes}

Participaron 32 trabajadores, divididos en dos grupos, asistentes a los talleres realizados entre octubre y noviembre de 2019. Eran profesionales de áreas como psicología, medicina, enfermería, trabajo social, gerontología y educación, así como operadores terapéuticos, los cuales tienen dentro de sus funciones la atención directa a los habitantes de calle de la ciudad.

Se estableció contacto con la Coordinación del Sistema de Atención al Habitante de Calle Adulto para la selección de la muestra intencional de los participantes clave [19], tratando de incluir diferentes voces en términos de su profesión, edad, género y experiencia profesional.

Se excluyeron aquellas personas que no dieron su consentimiento escrito para participar en la investigación.

\section{Proceso de sistematización, validación e interpretación de la información}

El proceso descriptivo y de interpretación se llevó a cabo a partir de la trascripción de las narraciones de los participantes; para esto, se hizo, inicialmente, un señalamiento por párrafos de frases cortas con sentido, de donde derivaron los primeros códigos descriptivos
(253), los cuales fueron utilizados para alimentar las categorías emergentes: "emociones" (153), "creencias" (21) y "actitudes" (79).

Tras el análisis categorial temático [20], se construyeron categorías conceptuales adscritas a la psicología social de las relaciones intergrupales [21], que fueron "prejuicio", "estereotipo" y "discriminación", y que se articulan al concepto de estigma propuesto por Goffman en 1963 [1]. Estas categorías permitieron discutir los hallazgos con la literatura disponible sobre el tema.

Ya que no se pudo alcanzar una saturación de los datos, dadas las características de la información (transcripciones de grabaciones de las sesiones de intervención), la validación cualitativa se efectuó mediante un proceso de revisión permanente y sistemático de la información.

Los hallazgos se llevaron a plenaria en el equipo de investigadores, con el objetivo de dar cumplimiento a los criterios de rigor en el análisis, en términos de auditabilidad/credibilidad [22]. Esto permitió un análisis interno sobre lo hallado, para obtener una visión de conjunto, de tal manera que las unidades de sentido estuvieran presentes en sus relaciones, semejanzas y diferencias [23], para luego triangular los hallazgos con la literatura científica disponible y las interpretaciones realizadas por los investigadores, asumiendo en todo momento una postura reflexiva por parte del equipo investigador.

El estudio cumplió con los principios éticos de respeto, beneficencia y justicia según la Declaración de Helsinki [24]. Se trató de una investigación sin riesgo, acogiéndose a la Resolución 8430 de 1993 [25], y previo aval del Comité de Ética en Investigación de la Universidad Pontificia Bolivariana de Medellín, en Acta 9 del 28 de mayo de 2018. Además, contamos con la firma del consentimiento informado por parte de cada participante.

\section{Resultados}

Los análisis realizados al material nos permitieron identificar temáticas emergentes según las voces de los participantes. Por eso, la presentación de los resultados se ordena en función de las emociones, creencias y actitudes, temas que están asociados conceptualmente al campo de la psicología social de las relaciones intergrupales.

\section{Emociones del personal asistencial hacia habitantes de calle que usan drogas}

Destacamos aquí los relatos de los participantes del grupo 1 y 2 (PGrupo 1 y PGrupo 2) acerca de las emociones sentidas hacia los habitantes de calle que consumen drogas. Algunos lograron identificar el lugar que tienen las emociones en su vínculo con los usuarios, diferenciando 
entre aquellas que facilitan la aceptación del otro en su estilo de vida y las que obstaculizan el logro de los objetivos en el ámbito laboral.

Una de las emociones que manifiestan los participantes es el asombro ante alguien que consume, porque este puede llegar a límites extremos, como apuñalarse a sí mismo o inyectarse en el lagrimal. Además, hay rabia ante situaciones en las que son agredidos o engañados por los usuarios, y si hay confrontación verbal o física y luego son amenazados, algunos expresan miedo:

[...] la verdad, ya me daba mucho miedo, y aunque sé que hasta el día de hoy él no ha agredido a nadie del personal, sí sentía demasiado temor y no le hacía tampoco consulta individual en el consultorio (PGrupo 1).

También nombran impaciencia ante los usuarios que no acatan las recomendaciones y se fugan de la institución para drogarse. Ante esto, aparece la ansiedad, como una manifestación centrada en pensamientos de preocupación por los usuarios, al desconocer dónde están, qué hacen y si han calmado los síntomas de abstinencia cuando no solicitan la ayuda del Sistema de Atención.

A esta última situación le agregan expresiones emocionales de tristeza, impotencia, decepción y desconfianza, marcadas por la frustración que atraviesa gran parte del tiempo el trabajo con esta población. Uno de los participantes indica:

Yo me sentí como impotente, porque sentí que era más como el deseo mío por su recuperación que el de él (PGrupo 2).

Y otro más agrega:

[...] uno lo que puede hacer es ofrecerle alternativas para que mejore sus condiciones de vida, pero no puede obligar a nadie a que las acepte, y esto es muy frustrante, porque predomina más el deseo de uno (PGrupo 2).

Según los participantes, las emociones negativas ya señaladas dan lugar a las resistencias ante los habitantes de calle que usan drogas, todo ello consolidado principalmente durante el trabajo en la institución, pues estos profesionales allí aprenden que hay algunas personas a las que no pueden ayudar, porque estas simulan una enfermedad para generar pesar, son conflictivas o recaen frecuentemente en el consumo de drogas, condicionando así el tipo de atención que los profesionales ofrecen.

Además de emociones negativas, sobresalen otras basadas en la confianza y en el reconocimiento de que los usuarios tienen sentimientos, sueños, ríen, sufren, pueden ser responsables y desear la recuperación. Describen historias de personas que se mantienen bien, están trabajando y agradecen por el acompañamiento, produciéndoles sentimientos de gratificación, esperanza y satisfacción.
También la empatía surge en los discursos, como base de las emociones positivas que orientan algunas de sus actividades y relaciones con los habitantes de calle, favoreciendo la labor y minimizando la frustración tan reiterada en sus opiniones. Así lo señalan:

Para mí es muy importante ponerse en el lugar del otro, ver a esa persona en su dimensión humana (PGrupo 2).

Agregan que detenerse a revisar sus emociones respecto a los usuarios les amplía su panorama sobre ellos:

Con este ejercicio, uno podría decir que se comprende un poco la situación, porque invita a ponerse en los zapatos del otro (PGrupo 1).

Estos resultados describen dos tendencias emocionales a nivel negativo y positivo, cada una con una funcionalidad, en términos de equilibrar la frustración propia del rol profesional y unas pretensiones personales para brindar ayuda a otra persona que se encuentra en condiciones extremas, como la habitabilidad en la calle y el consumo de SPA.

Destacamos que las emociones diversas se complejizan en el tiempo y se anudan a un conjunto de creencias que sustentan, en gran medida, las actitudes y los comportamientos que propician o no la estigmatización.

\section{Creencias de los participantes sobre los habitantes de calle usuarios de drogas}

La forma en que se relaciona el personal asistencial con los habitantes de calle que usan drogas está precedida por las creencias que aquellos han construido sobre estos o acerca del consumo. Estas creencias pueden relacionarse con la historia de vida del personal o pudieron haberse afianzado con el rol en el trabajo con habitantes de calle.

Las percepciones sobre estas personas varían entre considerarlos enfermos, incapaces de cambiar de opiniones, hasta creerlos seres irresponsables, que se aprovechan de su estado para mantener el consumo. Con relación a esto, surgen algunas ideas sobre la incapacidad que los usuarios tienen de aceptar que poseen una dificultad, que van a llegar a niveles crónicos de adicción, o entienden el consumo como un refugio ante un dolor físico o emocional.

Algunos de los participantes afirman que los habitantes de calle que usan drogas no tienen la capacidad para generar cambios en sus vidas, sin importar lo mal que estén. Así lo expresan:

Y ese impacto que me da, me pone a pensar en cómo hace un ser humano para llegar a esas condiciones y no hacer nada (PGrupo 1). 
Asumen que, ocasionalmente, el usuario puede querer dejar el consumo, pero no puede hacerlo, porque al ser una enfermedad, no solo depende de él. También piensan que la condición de consumo puede hacerles sentir vergüenza y les impide acercarse a los demás en condición de igualdad.

Otras creencias se soportan en los intentos por explicar qué lleva a los usuarios a la calle y al consumo, y su respuesta es que se da justamente por los consumos problemáticos sin control y no por una elección previa.

Los años de experiencia de trabajo con la población han generado un conjunto de ideas sobre el estado emocional de ese consumidor; de allí que uno de ellos exprese:

Durante estos años que llevo en el programa, he logrado detectar que ninguno está feliz por estar en esa situación de calle y de consumo de sustancias psicoactivas (PGrupo 2).

Algunos participantes identifican lo que la abstención del consumo genera y que por esta razón los usuarios retroceden en sus procesos, desaparecen algunos días y retornan a la institución, en muchas ocasiones, en estados delicados de salud.

Por otro lado, hay percepciones que dan cuenta de un rechazo del comportamiento de los usuarios, ya que los consideran aprovechados, desagradecidos o descarados. Según los participantes, se les debe asumir como sujetos de responsabilidad y no solo de derecho, lo cual puede asociarse con otras ideas respecto a ellos, como personas que se aprovechan de su vulnerabilidad porque están en la calle, situación que los hace exigentes en sus demandas, para que sean suplidas de manera inmediata; además, valoran poco lo que se hace por ellos. Esta idea se refuerza cuando los participantes señalan que a los usuarios se les da todo sin que ellos pongan de su parte, y afirman que no hay límites claros a nivel institucional, pues les satisfacen todas las exigencias sin un mínimo de espera, así como deben hacerlo otros grupos sociales para acceder a los servicios de salud, educación, justicia, entre otros. Por tanto, creen que el habitante de calle que usa drogas no hace mucho para ganarse las cosas.

Pese a este conjunto de creencias que asumen a los usuarios como irresponsables y pasivos, se destacan otras percepciones motivadas por intereses personales, en los que se reconocen procesos de identificación con la historia de los usuarios, porque es similar a la historia de vida de alguien cercano o a la propia. Al respecto, uno de los funcionarios expresa:

Cuando lo veía a él, pensé que podía hacerlo pensar lo mismo que yo, porque lo veía de mí misma estatura, color de piel, mi edad; eso me hizo querer estar ahí intentando, pero no. Ese caso siempre me ha impactado, no sé si porque me veo en él (PGrupo 1).
Esta posición devela las expectativas personales que se ponen en el proceso de los usuarios, quienes esperan, por ejemplo, una eliminación del consumo y no su reducción para considerar exitosa una intervención, lo que explicaría la aparición recurrente de emociones propias a la frustración, como fue señalado anteriormente.

Otro asunto que se pone de manifiesto cuando el funcionario está en el proceso de acompañamiento son los retos que se imponen para que el usuario salga de la situación en la que se encuentra. Para ello, buscan estrategias, como insistir sin mostrar explícitamente este interés, o convertir su labor en un reto personal. Al respecto mencionan:

Entonces, yo me di como la tarea de acercarme como para generar empatía, pero él tampoco como que se abre para dejarse ayudar. Entonces, cuando nosotros lo levantábamos, nos sacaba cuchillo y nos correteaba por todo; pero en mí seguía la intención y yo me lo tomé como a manera de meta; yo pensaba: "Me lo tengo que llevar para una unidad hospitalaria, así sea para que le hagan alguna cosa", y cierto día pasé por el lado de él y lo saludé sin decirle nada, y seguí; entonces, él me llamó y me dijo que sí quería la ayuda, y entonces, yo de una activé la ruta y lo llevamos a San Cristóbal, y yo feliz, porque estaba logrando mi meta; pero imagínese que allí se evade y quedo con ese sinsabor (PGrupo 1).

Resaltamos otras ideas sobre habitar la calle o usar drogas como una elección de vida:

$\mathrm{Si}$ el otro quiere fumar marihuana en su proyecto de vida, eso es escandalizante para muchos profesionales y no se acepta que es la elección del otro (PGrupo 1).

Y comenta un participante que alguien lo interpela al respecto:

[...] "profe, ¿es que a usted quién le dijo que yo quiero dejar de ser habitante de calle?" (PGrupo 1).

Con estas creencias, los profesionales intentan explicar ambos fenómenos, siendo la última un desafío, porque va en contravía del ideal del acompañamiento para abandonar la calle y las drogas, cuestionándose el hecho de no poder realizar un acompañamiento acorde con las necesidades de los usuarios, para tener menos frustraciones.

En este sentido, algunos de los participantes hacen un llamado a tener más apertura en las intervenciones, incluso en el lenguaje utilizado para referirse a ellos, siendo esto un elemento que les orienta en sus actividades de acompañamiento.

Además, señalan que entender la magnitud de la adicción favorece una creencia con menos prejuicios respecto al habitante de calle consumidor de drogas, en comparación con otras personas que no tienen ese conocimiento, y destacan la necesidad de diferenciar entre el 
consumo como elección personal y la expectativa que cada profesional tiene al respecto.

Por otra parte, varios de los profesionales señalan que buscan cambiar la forma de percibir al habitante de calle y reconocerlo como una persona con derechos, digna de atención y oportunidades, y aceptar que a pesar de la perseverancia que deben tener para lograr resultados, vale la pena hacerlo, especialmente si se tiene en cuenta que

También hay muchos usuarios que no quieren dejar de consumir por completo; quisieran dejar algunas drogas que les generan problemas y continuar con el consumo de otras (PGrupo 2).

\section{Actitudes del personal asistencial hacia los habitantes de calle}

Entre las actitudes que manifiestan los funcionarios hacia el habitante de calle consumidor de SPA, se evidencian algunas asociadas a la protección y disposición de ayuda para lograr que las personas a las que acompañan dejen de consumir drogas o cambien su condición. Así lo nombran:

Uno llega al sistema con esa moral, con esas ganas de ayudar a toda esa gente. Uno cree que va a salvar el mundo, que le van a copiar [le van a hacer caso] a uno, que los va a sacar de las drogas, pero uno está muy equivocado (PGrupo 1).

El interés por ayudar, así el otro se muestre reacio a recibir ese apoyo, se debate entre quienes, como se mencionó anteriormente, lo asumen como un objetivo primordial y los otros que lo conciben como una elección de vida:

Uno no pretende que ellos cambien, pues si eso es lo que ellos quieren, pues qué se va a hacer, uno está ahí para ayudarles cuando pueda (PGrupo 1).

Otra actitud identificada se relaciona con posturas evasivas que nombran los participantes para liberarse temporalmente de lo que ellos denominan "cargas emocionales" que alimentan la frustración, pues manifiestan que, en su hacer profesional, cuando no logran alcanzar los objetivos con la población o cuando se presentan casos "complejos", recurren a la evasiva justificada, por ejemplo, en la creencia de que el habitante de calle es irresponsable y "aprovechado". De allí que se presenten expresiones como:

Yo me he encontrado con casos tan complejos y que uno ve ya tan llevados [en muy malas condiciones], así uno haya hecho hasta lo imposible, que yo mejor dejo así e intento olvidar hasta el caso. Uno se frustra tanto, que prefiere ya no hacer nada (PGrupo 2).

Lo anterior también ha generado que algunos funcionarios tengan una actitud de prevención ante los usuarios, refiriendo que estos, además de no asumir responsabilidades, mienten, son agresivos o se aprovechan de quienes les brindan ayuda, en este caso, del personal asistencial. Por ejemplo, un participante afirma:

A mí lo que me pasa es que muchas veces [me] mantengo prevenido, ya uno no sabe si creerles, y es que incluso muchos son agresivos con nosotros, sobre todo con los educadores. Se aprovechan y quieren que uno les de todo, los ayude, pero ellos no responden, y si uno no hace lo que quieren, es como si nunca los hubiera ayudado (PGrupo 2).

Algunos participantes manifiestan que han intervenido situaciones específicas cuando han sido agredidos por usuarios. En algunos casos, han tomado medidas apresuradas en momentos donde pierden el control debido a una rabia, respondiendo con actitudes agresivas:

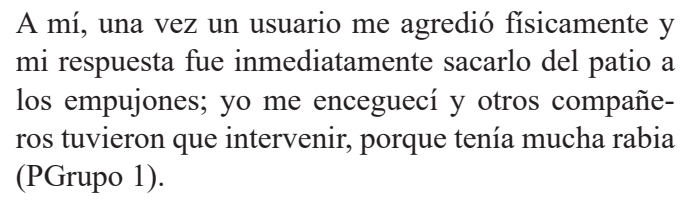

A lo anterior, se suman las actitudes de desconfianza por parte de los funcionarios, quienes refieren constantemente que las acciones de los usuarios, en las que se les dificulta asumir responsabilidades y cumplir compromisos, han generado que las relaciones se basen en cuestionamientos y sanciones frecuentes.

Finalmente, y como complemento a las actitudes negativas mencionadas, es importante nombrar la insistencia de los participantes en la investigación en el acompañamiento como una actitud de perseverancia, pese a la frustración generada por el incumplimiento de los objetivos propuestos y de las expectativas planteadas. Se sienten responsables del bienestar de los usuarios y suponen que la persistencia les permitirá lograr avances en el proceso. Uno de ellos señala:

[...] yo siento que si uno no es persistente, no logra nada. Uno ve que ellos logran avanzar cuando uno está ahí de manera constante y así sean avances pequeños, se siente esa satisfacción de saber que uno aportó algo (PGrupo 1).

\section{Discusión}

Las alusiones a las emociones, las creencias y las actitudes comportamentales de los funcionarios hacia los usuarios tienen su correspondencia con el fenómeno del estigma en general y en específico con los prejuicios, los estereotipos y la discriminación, respectivamente [26]. Y se trata de una relación uno a uno, porque se entiende acá el prejuicio como las predisposiciones emocionales, habitualmente negativas, con respecto a miembros del grupo cuyas características están sujetas a creencias es- 
tereotipadas [21]. Por su parte, el estereotipo se refiere al conjunto de ideas, creencias o concepciones positivas y negativas, pero generalmente erróneas, que la mayoría de la población mantiene en relación con un determinado grupo social, y que condicionan la valoración de muchas de las características y conductas de los miembros de dicho grupo, construyendo una "identidad deteriorada" a partir de una característica evaluada [26]. Y, finalmente, la discriminación es el comportamiento que implica la propensión a desarrollar acciones negativas, habitualmente medidas en términos de distancia social hacia los miembros del grupo vinculado con un estereotipo negativo y que representan la vulneración de los derechos de la persona o colectivos que poseen un rasgo considerado como indeseable. Los aportes en este campo temático de las relaciones intergrupales se han referido al antagonismo entre miembros de grupos sociales y étnicos diversos [21], pero nosotros nos centramos en las relaciones entre el personal asistencial y los habitantes de calle que consumen drogas.

En este orden de ideas, destacamos que el prejuicio, en su connotación emocional negativa [21], recurrente entre los participantes de este estudio, coincide con otras investigaciones centradas en profesionales de la atención primaria y especializada del sector salud, en las cuales se reitera que a dicho prejuicio le anteceden ideas negativas sobre la droga en sí misma, recayendo así la responsabilidad exclusivamente en la persona consumidora, sin que se tengan en cuenta otros asuntos contextuales que también están implicados, por ejemplo, la responsabilidad del Estado o la precariedad del sistema sanitario[13,27].

Ahora bien, la frustración del profesional, reiterada por nuestros participantes, se asocia a la hipótesis de la frustración-agresión de Dollard et al. propuesta en 1939 [citado en 21, p. 9], que explica la aparición del estigma y las reacciones negativas sustentadas en una frustración previa respecto al exogrupo. Para los participantes del estudio, esto se evidencia en las actitudes de evasión y prevención fundadas en las creencias negativas de aquel que habita las calles y usa drogas con poca capacidad para salir de dicha situación, lo que propicia la discriminación hacia los usuarios y genera dificultades en la atención a la persona adicta [27].

Lo anterior también puede favorecer un retraso en la búsqueda de tratamiento por parte de PUD, incluso para los sujetos que están motivados en hacerlo, lo que influye en la consecución de los objetivos del mismo [5] y agrava los problemas de salud que pudieran presentar las personas que quieran buscar ayuda [13]. Por eso, es preciso tener presente el impacto que tendrían las ideas y los afectos positivos en los procesos de acompañamiento, como se identificó en las creencias del habitante de calle y consumidor como un ser humano con metas y sueños, que comete errores y que está dispuesto a asumir consecuencias, lo que contribuye a una actitud de perseverancia y a una satisfacción con el deber cumplido, así en algunos casos haya una recaída, no se logre una abstinencia total o la persona se mantenga en situación de calle.

En cuanto a la abstinencia, es preciso recordar que, en el siglo xx, tomó fuerza la idea acerca de la necesidad de una vida libre de drogas; y puesto que solo quienes se abstenían del consumo eran considerados como personas "normales", quienes usaban drogas empezaron a ser etiquetados y estigmatizados como "enfermos", "desviados" o "degenerados". Esto condujo a la fundamentación de los abordajes punitivos, orientados a la abstinencia total [28], desconociendo que hay otros objetivos intermedios que pueden ser más significativos que la abstinencia, como la reducción del daño y la marginalidad, la disminución de la mortalidad, la mejoría de la salud física y mental, la disminución del deseo de consumir (craving), la comunicación efectiva, el manejo del tiempo libre, las relaciones afectuosas, el control de contingencias, la habilidades sociales para la vida, la disminución de su participación en actividades delictivas, la espiritualidad y la construcción del proyecto de vida, entre otros [29,30].

Cabe señalar que la frustración que se genera en el personal asistencial porque los usuarios no quieren cambiar su situación hace que los profesionales vean como la única alternativa la desintoxicación y reeducación para alcanzar la abstinencia total, convirtiéndose la labor del terapeuta en persuasiva y moralizadora [31], o transgresora de los límites entre lo público y lo privado, con el pretexto de proteger a los usuarios [32].

Por otro lado, y coincidiendo con un estudio en Brasil [33] y otro en Colombia [13], los participantes de nuestro estudio centran sus percepciones negativas en la droga, sin tener en cuenta las condiciones estructurales y sociales que la han caracterizado en el contexto de estos países latinoamericanos, con lo que se refuerza la idea de la responsabilidad exclusiva del individuo que carece de la capacidad para tomar decisiones y a quien debería protegerse de las drogas porque son nocivas [34]. De allí la importancia de diferenciar entre una estigmatización y las actitudes negativas hacia un tipo particular de droga, por ejemplo, la marihuana o la cocaína [35], o hacia un grupo social específico que consume, como mujeres embarazadas o personas pobres [36].

Finalmente, entendemos que los aspectos cognitivos y emocionales negativos de la estigmatización tienen generalmente una correspondencia con un componente comportamental también negativo referido a prácticas concretas de rechazo y exclusión social [21], porque son procesos interrelacionados, lo cual ha sido consistente con afirmaciones de varias investigaciones, en particular con personal sanitario $[5,13,37]$, en las que se insiste en la necesidad de reducir el estigma para facilitar el acce- 
so a la salud y demás servicios a los que todos tenemos derecho $[12,38]$.

Las limitaciones de este trabajo se centran en el contexto de los participantes, pues el personal asistencial labora exclusivamente con habitantes de calle de Medellín; por tanto, la referencia al estigma se circunscribe a ese escenario de trabajo institucional.

\section{Conclusiones}

Los procesos de acompañamiento llevados a cabo por el personal asistencial han sido permeados por las emociones que pueden surgir del vínculo que se establece con los usuarios, de las experiencias de vida previa o por las que transitan en el entorno laboral. Aunque prevalecen las emociones negativas, como rabia, tristeza, temor, frustración, entre otras, también sobresalen unas basadas en la confianza y en el reconocimiento del otro, fundado en el interés por ayudarlo a salir del contexto de la calle y del uso de drogas, que produce un sentimiento de gratificación y satisfacción. Esto último facilita la empatía evidenciada en los discursos de los participantes como una forma de orientar las actividades y relaciones con los consumidores de drogas y habitantes de calle, lo cual influye en la intervención y en el sentimiento de frustración en los profesionales.

Finalmente, concluimos que la coherencia entre las emociones, creencias y actitudes hacia el habitante de calle se fundamenta en unas comprensiones sobre el objeto "droga", unido al fenómeno de la habitancia en calle y de la estigmatización en sí misma. Así, la frustración en el trabajo que este grupo de personas dice sentir se mantiene cuando el ideal del acompañamiento se centra en el abandono absoluto de la droga y de la calle, como si el objetivo fuera la droga y no la persona misma y las otras situaciones que la rodean. Esto involucra el reconocimiento de la condición humana, tanto del profesional como de quien consume drogas y habita la calle, pues allí están las expectativas individuales de ambas partes, correspondientes a sus realidades subjetivas, familiares y sociales.

Las creencias y percepciones construidas por los participantes sobre el consumo y los usuarios de drogas anteponen el modo de establecer vínculo con esta población. De acuerdo con esto, se concibe a los consumidores de una forma subjetiva, en tanto es mediada por la construcción social en torno al fenómeno, que genera algunas discrepancias entre el deseo de los profesionales y los intereses de los usuarios. Esta concepción subjetiva también se basa en el desconocimiento de un contexto social e histórico más amplio, que explicaría el lugar que tienen las emociones, creencias y actitudes negativas en los procesos de acompañamiento y que propician el estigma hacia este grupo de personas.
Subrayamos con esto la importancia de reflexionar acerca del estigma en el personal asistencial en su vínculo permanente con usuarios de drogas, puesto que por sí solo esto no reduce el conjunto de creencias, emociones y conductas negativas. Por eso, varios serían los frentes de acción para reducir el estigma hacia habitantes de calle que usan drogas: por un lado, las prácticas educativas dirigidas a cualquier grupo de personas [12] y, por otro, la permanente exigencia de cambios en las políticas públicas, que debieran basarse más en el conocimiento científico y menos en postulados morales, para que de este modo contribuyan realmente al mejoramiento de las condiciones de vida de las comunidades y en especial del sistema social y sanitario [13].

Con respecto a las actitudes de los funcionarios hacia el habitante de calle que usa drogas, se puede concluir que aquellas aparecen en concordancia con la carga emocional, pero se manifiestan de manera ambivalente según las ideas previas que se reconfiguran a partir de las experiencias personales y profesionales, de su formación y su capacidad reflexiva.

Finalmente, destacamos que el estudio tiene límites en sus alcances, en cuanto a la posibilidad de generalización para todos los grupos de profesionales que trabajan con habitantes de calle que usan drogas en otras ciudades del país. Y su énfasis en el nivel descriptivo del análisis facilita el planteamiento de nuevos interrogantes, mas no la comprensión del fenómeno del estigma hacia personas que habitan la calle y consumen drogas.

\section{Agradecimientos}

Agradecemos a la Secretaría de Inclusión Social, Familia y Derechos Humanos de la Alcaldía de Medellín y a su Sistema de Atención al Habitante de Calle Adulto; a la "Estrategia de Sostenibilidad Grupos de Investigación 2018-2019" del Comité para el Desarrollo de la Investigación (CODI) de la Universidad de Antioquia, y a nuestros participantes, por permitirnos hacer la investigación.

\section{Declaración de fuente de financiación}

Convocatoria UPB Innova 2018 de la Universidad Pontificia Bolivariana (UPB), y de la "Estrategia de Sostenibilidad Grupos de Investigación 2018-2019" del CODI de la Universidad de Antioquia, la Universidad Católica Luis Amigó y la Corporación Surgir.

\section{Declaración de conflictos de intereses}

Los autores manifestamos que no hay conflictos de intereses. 


\section{Declaración de responsabilidad}

Los autores declaramos que el diseño del proyecto fue iniciativa del profesor Andrés Felipe Tirado. Y referimos que todo el equipo hizo parte del proceso de recolección y análisis de la información, y contribuimos con la escritura del artículo, además de su aprobación final, de manera que cada uno puede dar cuenta de los principales argumentos y afirmaciones presentadas en el manuscrito. Además, las instituciones de afiliación y los financiadores no asumen responsabilidad sobre el contenido del texto.

\section{Declaración de contribución por autores}

Maricelly Gómez Vargas: diseño del proyecto, recolección, análisis y discusión de la información, escritura del artículo y aprobación final del artículo.

Eliana María Hernández Ramírez: diseño del proyecto, recolección, análisis y discusión de la información, escritura del artículo y aprobación final del artículo.

María Jimena Osorio Salazar: recolección, análisis y discusión de la información, escritura del artículo y aprobación final del artículo.

Andrés Felipe Tirado Otálvaro: diseño del proyecto, recolección, análisis y discusión de la información, escritura del artículo y aprobación final del artículo.

José Silverio Espinal Bedoya: diseño del proyecto, recolección, análisis y discusión de la información, escritura del artículo y aprobación final del artículo.

Daniel Ricardo Zaraza Morales: recolección, análisis y discusión de la información, escritura del artículo y aprobación final del artículo.

Lucas Dávila Cañas: diseño del proyecto, recolección, análisis y discusión de la información, escritura del artículo y aprobación final del artículo.

Luz Merly Saldarriaga Agudelo: recolección, análisis y discusión de la información, escritura del artículo y aprobación final del artículo.

\section{Referencias}

1. Goffman E. Stigma, notes on the management of spoiled identity. New Yersey: Prentice Hall Englewood Cliffs, editor; 1963.

2. Link BG, Phelan JC. Conceptualizing stigma. Annu Rev Sociol. 2001;(27):363-85. DOI: https://doi.org/10.1146/annurev. soc. 27.1 .363

3. Campo-Arias A, Herazo E. El complejo estigma-discriminación asociado a trastorno mental como factor de riesgo de suicidio. Rev Colomb Psiquiatr. 2015;4(44):243-50. DoI: https://doi.org/10.1016/j.rcp.2015.04.003
4. Pescosolido BA, Martin J. The stigma complex. Annu Rev Sociol. 2015;41:87-116. DoI: https://doi.org/10.1146/annurevsoc-071312-145702

5. Ronzani T, Higgins-Biddle J, Furtado EF. Stigmatization of alcohol and other drug users by primary care providers in Southeast Brazil. Soc Sci Med. 2009;69(7):1080-4. DoI: https://doi. org/10.1016/j.socscimed.2009.07.026

6. Vásquez A. El estigma sobre la drogodependencia y su relación con la accesibilidad a servicios de salud. Psicologías. [internet] 2009;0;1-7. [citado 2021 abr. 10]. Disponible en: https://revistas. upr.edu/index.php/psicologias/article/view/17199

7. Cassiani-Miranda CA, Campo-Arias A, Herazo E. Trastornos por consumo de sustancias: entre las inequidades $\mathrm{y}$ el estigma. Duazary. 2019;16(2):271-80. DoI: https://doi. org/10.21676/2389783X.2751

8. Colombia, Ministerio de Salud y Protección Social. Oficina de Promoción Social, Grupo de Gestión Integral en Promoción Social. Política pública social para habitantes de calle [internet]. 2018 [citado 2020 sep. 24]. Disponible en: https://www.minsalud. gov.co/sites/rid/Lists/BibliotecaDigital/RIDE/DE/PS/politicapublica-social-habitante-de-calle.pdf

9. Red Iberoamericana de Organizaciones No Gubernamentales que Trabajan en Drogas y Adicciones (RIOD). Estigma, consumo de drogas y adicciones. Conceptos, implicaciones y recomendaciones [internet]. Madrid; 2019. 57 p. [citado 2020 sep. 24]. Disponible en: https://riod.org/wp-content/uploads/2019/06/ESTIGMACONSUMO-DE-DROGAS-Y-ADICCIONES.pdf

10. Centro de Estudios de Opinión Universidad de Antioquia. Caracterización de habitantes de calle de la ciudad de Medellín. Medellín: Alcaldía de Medellín, Secretaría de Inclusión Social y Familia, editor; 2014.

11. Departamento Administrativo Nacional de Estadística. Censo habitantes de la calle 2019. Resultados Medellín y Área Metropolitana [internet]. 2019 [citado 2020 oct. 4]. Disponible en: https://www.dane.gov.co/files/investigaciones/boletines/censohabitantes-calle/medellin-am-2019.pdf

12. Ronzani T, Noto A, Silveira P. Reduzindo o estigma entre usuários de drogas. Guia para profissionais e gestores. Juiz de Fora: UFJF, editor; 2014.

13. Tirado AF, Calderón G, Restrepo S, et al. Estigma social de profesionales de la salud hacia personas que usan drogas. Psicol em Pesqui. 2019;13(1):22-32. doi: https://doi.org/10.34019/19821247.2019.v13.23863

14. Pascual M, Pascual F. El estigma en la persona adicta. Adicciones [internet]. 2017;29(4):223-6. [citado 2021 abr. 10]. Disponible en: https:/www.adicciones.es/index.php/adicciones/article/viewFile/1038/901

15. Sánchez N. ¿Disminuye la calidad asistencial de los pacientes drogodependientes debido al estigma o actitudes de los profesionales sanitarios? [trabajo de fin de grado]. [Islas Baleares]: Universitat de les Illes Balears [internet]; 2020 [citado 2021 abr. 10]. Disponible en: https://dspace.uib.es/xmlui/bitstream/handle/11201/153375/ Sanchez_Bestard_Neus.pdf?sequence $=1 \&$ isAllowed $=\mathrm{y}$

16. Vásquez A, Stolkiner A. Procesos de estigma y exclusión en salud. Articulaciones entre estigmatización, derechos ciudadanos, uso de drogas y drogodependencia. Anu Investig. [internet]. 2009 [citado 2021 abr. 10]; 16:295-303. Disponible en: https://www. redalyc.org/pdf/3691/369139945027.pdf

17. Vásquez A. Barreras de accesibilidad de drogadependientes a servicios de salud. El caso de la ciudad de Buenos Aires [internet]. 2013. [citado 2021 abr. 10]. Disponible en: https://www.aacademica.org/000-054/310 
18. Rada Cadenas DM. El rigor en la investigación cualitativa: técnicas de análisis, credibilidad, transferibilidad y confirmabilidad. Sinop Educ. [internet] 2007 [citado 2020 sep. 15]; 7(1):17-26. Disponible en: http://revistas.upel.digital/index.php/sinopsis_ educativa/article/view/3539

19. Martínez-Salgado C. El muestreo en investigación cualitativa: principios básicos y algunas controversias. Ciênc. Saúde Colet. 2012;17(3):613-9. DoI: https://doi.org/10.1590/S141381232012000300006

20. Vázquez-Sixto F. El análisis de contenido temático. En: Objetivos y medios en la investigación psicosocial. (Documento de trabajo). (pp.47-70). Universitat Autónoma de Barcelona; 1996.

21. Smith-Castro V. La psicología social de las relaciones intergrupales: modelos e hipótesis. Actual en Psicol. [internet] 2006 [citado 2020 sep. 15]; 20(107):45-71. Disponible en: http:// pepsic.bvsalud.org/pdf/apsi/v20n107/v20n107a03.pdf

22. Creswell JW, Miller DL. Determining validity in qualitative inquiry. Theory Pract. 2000;39(3):124-30. Dor: https://doi. org/10.1207/s15430421tip3903_2

23. Navarrete JVM. Problemas centrales del análisis de datos cualitativos. Rev Latinoam Metodol Investig Soc. [internet] 2011 [citado 2020 oct. 2]; 1(1):47-60. Disponible en: http://relmis.com.ar/ojs/ index.php/relmis/article/view/43/46

24. Word Medical Association. wMA Declaration of Helsinki. Ethical principles for medical research involving human subjects. [internet] 2013 [citado 2020 oct. 2]. Disponible en: https://www. wma.net/policies-post/wma-declaration-of-helsinki-ethicalprinciples-for-medical-research-involving-human-subjects/

25. Colombia, Ministerio de Salud y Protección Social. Resolución 008430, por la cual se establecen las normas científicas, técnicas y administrativas para la investigación en salud (1993 octubre 4).

26. Rengel D. La construcción social del "otro". Estigma, prejuicio e identidad en drogodependientes y enfermos de sida. Gaz Antropol. [internet]. 2005 [citado 2020 sep. 14]; 21:1-14. Disponible en: https://www.ugr.es/ pwlac/G21_25Daniel_Rengel_Morales.html

27. Paiva FS, Ferreira ML, Martins MZF, et al. A percepção profissional e comunitária sobre a reinserção social dos usuários de drogas. Psicol Soc. 2014;26(3):696-706. DoI: http://dx.doi.org/10.1590/ S0102-71822014000300018

28. Martínez DP, Pallarés J. Riesgos, daños y placeres. En: Martínez DP, Pallarés J, editores. De riesgos y placeres. Manual para entender las drogas. Lleida: Milenio; 2013. pp. 23-36.
29. Giraldo-Ferrer, LF., Velásquez-Tirado, JD., Restrepo-Medrano, JC., Tirado-Otálvaro A. Uso de instrumentos clínicos para la evaluación de pacientes y resultados de tratamiento en trastornos por uso de sustancias (TUS). Rev Fac Med. 2016;64(4):749-59. DoI: http://dx.doi.org/10.15446/revfacmed.v64n4.54697

30. Romaní O. Reducción de daños y control social. ¿De qué estamos hablando? En: Martínez DP, Pallarés J, editores. De riesgos y placeres. Manual para entender las drogas. Lleida: Milenio; 2013. pp. 103-16.

31. Lobos M. Políticas públicas para el tratamiento y rehabilitación de personas con consumo problemático de drogas [tesis pregrado]. [Santiago de Chile]: Universidad de Chile [internet]; 2012 [citado 2020 oct. 30]. Disponible en: http://repositorio.uchile.cl/ handle/2250/117084

32. Capalbo M. Prohibición de las drogas psicoactivas (ilegales) y neopunitivismo. Rev Redbioética Unesco. [internet] 2013 [citado 2020 oct. 15]; 4(7):35-44. Disponible en: https://redbioetica.com. ar/wp-content/uploads/2018/11/Art3-CapalboR7.pdf

33. Duarte Bard N, Antunes B, Moraes Roos C, et al. El estigma y el prejuicio: la experiencia de los consumidores de crack. Rev Latino-Am Enfermagem. 2016;24:1-7. DoI: https://doi. org/10.1590/1518-8345.0852.2680

34. Martinón Quintero R. La importancia de las ideas en las políticas públicas de los sistemas democráticos. El caso de la política de lucha contra las drogas [internet]; 2005. [citado 2020 oct. 2]. Disponible en: https://aecpa.es/files/congress/7/actas/area03/GT08/ MARTINON-QUINTERO-Ruth(UC3M).pdf

35. Delgado C, Brands B. Actitudes hacia las personas con usos problemáticos de drogas en la ciudad de Loja, Ecuador. Texto Context Enferm. 2019;28:1-13. Dor: https://doi.org/10.1590/1980265x-tce-cicad-11-11

36. Diez M, Pawlowicz M, Vissicchio F, et al. Entre la invisibilidad y el estigma: consumo de sustancias psicoactivas en mujeres embarazadas y puérperas de tres hospitales generales de Argentina. Salud Colect. 2020;16:1-19. DoI: https://doi.org/10.18294/ sc. 2020.2509

37. Kulesza M, Matsuda M, Ramirez JJ, et al. Towards greater understanding of addiction stigma: Intersectionality with race/ethnicity and gender. Drug Alcohol Depend. 2016;169:85-91. DoI: https://doi.org/10.1016/j.drugalcdep.2016.10.020

38. Mendez K, Ronzani T, Santana F. Se essa rua falasse: uma análise sobre estigma, pobreza e uso de drogas nas trajetórias de sujeitos em situação de rua. Juiz de Fora: UFJF, editor; 2019. 